\title{
Mathematical Model of Forecasting Laser Marking Experiment Results
}

\author{
Pavels Narica Artis Teilans Lyubomir Lazov Pavels Cacivkins Edmunds Teirumnieks \\ Faculty of Engineering, Rezekne Academy of Technologies, Latvia, \\ laserarta.lv
}

\begin{abstract}
Method allows for modelling of the anticipatory results of colour laser marking experiments. The process of calculating expected results takes into consideration the construction specifics of laser system being used and displays the results in compact form of a set of parameter matrices that have their values conditionally formatted as colour maps for easy identification of complex patterns. The complete set of all the related parameter matrices, both technical and derived, as well as the specific relations between them form the mathematical model of forecasting laser marking experiment results. Because the mathematical model is implemented within spreadsheet processor, it can be instantiated multiple times for any number of experiments.
\end{abstract}

Keywords: mathematical model, modelling method, laser system construction specifics, visualization of parameters, matrices, experiments, colour laser marking

\section{Introduction}

The main goal of this research is to simplify the process of forecasting and visualizing the distribution of values of experimental laser parameters.

Laser systems are widely used and provide some spectacular capabilities in many different fields. However lasers are complicated systems and operating a laser system requires a very good understanding of how lasing is actually done and how it can affect surroundings.

One case of common use of laser systems is colour laser marking on metal. Pulsed fiber lasers are usually used for producing markings of different colours on metal workpiece. Such laser systems are rather small in size, very precise, relatively inexpensive, and provide easy to use computer software for plotting laser marking elements. When plotting different elements in marking software, different technical laser parameters can be assigned to them in order to produce different marked colours (Laasko et al, 2008; Antonczak et al, 2014; Veiko et al, 2016; Qia et al, 2003; Antonczak et al, 2013; Amara et al, 2015; Lehmuskero et al, 2010; Veiko et al, 2014).
In many cases while operator is testing the laser system for its marking capabilities he/she marks some matrices of different combinations of laser's technical or other physical parameters. While this is logical approach, in most such cases it has serious issues, because the construction of laser system may behave differently under different set of parameters and thus produce unexpected or difficult to interpret marking results.

Because metal workpieces used for laser marking tests usually have rectangular flat two-dimensional surface (Figure 1), plotting on them rectangular matrices of rectangular elements is very efficient. This way an operator can assign for all the rows of such matrix some distinct technical or other test parameter and then assign all columns some other distinct test parameter while at the same time varying linear or logarithmic values of both assigned test parameters for each row and each column.

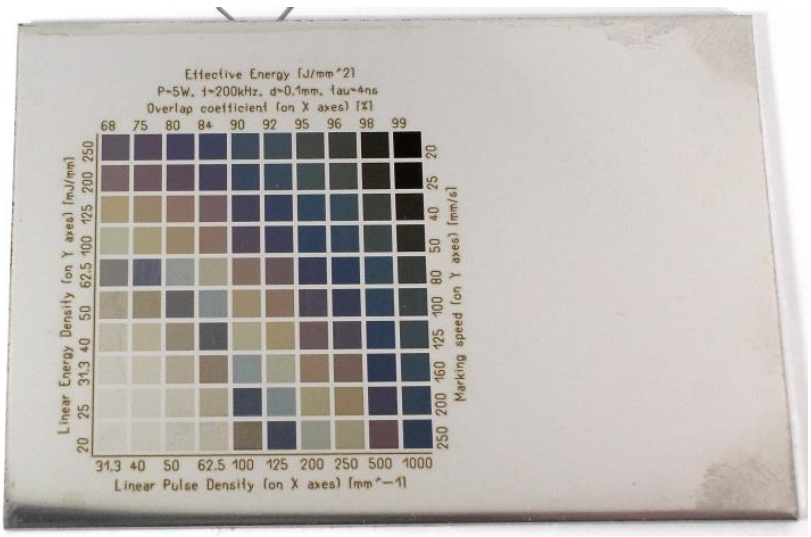

Figure 1. Experimentally produced sample marking colours on stainless steel workpiece.

When both dynamic test parameters are assigned to rows and columns of the matrix, all remaining parameters for all matrix elements are set to have some constant value. Such plotted matrix is then marked and can be further analyzed. This way one should be able to see how varying values of only two test parameters affect the produced marking colours. Though, as it was mentioned above, in some cases the construction of laser system itself can indirectly affect some additional parameters thus making analysis of marking colours against selected dynamic test parameters of marked matrix much more difficult and unintuitive. 
When output marking samples are analyzed, the corresponding output colours are usually mapped against technical and other physical parameters set dynamically before marking test as well as other constant technical laser parameters and parameters related to the nature of marking process itself. This approach helps one better see which parameters cause marking colours to change. Yet such mappings usually are in form of linear flat tables requiring lots of rows for each experimentally marked colour and columns for listing all parameters to display all the necessary data. Within such table it may become very hard for one to efficiently identify important parameters that actually affect the formation of marked colour.

Finally, it is common practice to produce marking experiment first and then to analyze resulting colours against parameters afterwards. Such practice can often result in regions within marked test matrix that do not have many distinct and quality colours in them.

The present method allows for modelling colour laser marking experiments while considering the construction specifics of a laser system. In particular, it is a novel method that deploys built-in spreadsheet processor's features in order to automatically generate informative numeric data matrices and their visualizations by means of conditional formatting (Figure 2, Figure 3).

\begin{tabular}{|c|c|c|c|c|c|c|c|c|c|}
\hline & \multicolumn{5}{|c|}{$\theta\left[\mathrm{J} / \mathrm{mm}^{\wedge}-2\right]$} & nsity & & & \\
\hline 3.1 & 4 & 5 & 6.3 & 10 & 13 & 20 & 25 & 50 & 100 \\
\hline 2.5 & 3.2 & 4 & 5 & 8 & 10 & 16 & 20 & 40 & 80 \\
\hline 1.6 & 2 & 2.5 & 3.1 & 5 & 6.3 & 10 & 13 & 25 & 50 \\
\hline 1.3 & 1.6 & 2 & 2.5 & 4 & 5 & 8 & 10 & 20 & 40 \\
\hline 0.8 & 1 & 1.3 & 1.6 & 2.5 & 3.1 & 5 & 6.3 & 13 & 25 \\
\hline 0.6 & 0.8 & 1 & 1.3 & 2 & 2.5 & 4 & 5 & 10 & 20 \\
\hline 0.5 & 0.6 & 0.8 & 1 & 1.6 & 2 & 3.2 & 4 & 8 & 16 \\
\hline 0.4 & 0.5 & 0.6 & 0.8 & 1.3 & 1.6 & 2.5 & 3.1 & 6.3 & 13 \\
\hline 0.3 & 0.4 & 0.5 & 0.6 & 1 & 1.3 & 2 & 2.5 & 5 & 10 \\
\hline 0.3 & 0.3 & 0.4 & 0.5 & 0.8 & 1 & 1.6 & 2 & 4 & 8 \\
\hline
\end{tabular}

Figure 2. Distribution of planar energy densities for sample.

\section{Materials and methods}

Experiments were carried out using PowerLine F-20 Varia series pulsed fiber laser system produced by Rofin-Sinar Laser GmbH. It emits photons of wavelength equal to $1064 \mathrm{~nm}$, has maximum average lasing power of $20 \mathrm{~W}$, pulse repetition rate of 2-1000 $\mathrm{kHz}$, adjustable pulse width of 4-200 ns. The stainless steel sample used for colour laser marking was 4301 189E $2 \mathrm{R}$.

Table 1 provides information used by current implementation of the mathematical model. It uses such common technical parameters as $f$ for pulse repetition rate, $v$ for scanning speed, $P$ for average lasing power, $\Delta x$ for line step (distance between two lines), $x$ and $y$ for width and length of individual rectangular marking element (Bliedtner et al, 2013; Eichler, 1998).

Table 1. Formulas used within proposed mathematical model as an example.

\begin{tabular}{|l|l|l|}
\hline \multicolumn{1}{|c|}{ Description } & \multicolumn{1}{c|}{ Formula } & Base unit \\
\hline Pulse overlap (linear pulse density) & $N_{y}=\frac{f}{v}$ & {$\left[\mathrm{~mm}^{-1}\right]$} \\
\hline Line overlap (linear line density) & $N_{x}=\frac{1}{\Delta x}$ & {$\left[\mathrm{~mm}^{-1}\right]$} \\
\hline Pulse energy & $E_{P}=\frac{P}{f}$ & {$[\mu J]$} \\
\hline Planar pulse density & $N=N_{y} \cdot N_{x}$ & {$\left[\mathrm{~mm}^{-2}\right]$} \\
\hline Planar energy denstiy & $\Theta=E_{P} \cdot N_{y} \cdot N_{x}$ & {$\left[\mathrm{~J} / \mathrm{mm}^{-2}\right]$} \\
\hline Total energy delivered to element & $E=\Theta \cdot y \cdot x$ & {$[\mathrm{~J}]$} \\
\hline Total marking time of element & $t=\frac{N_{x} \cdot x \cdot y}{v}$ & {$[s]$} \\
\hline
\end{tabular}

The method of modelling colour laser marking experiments takes into consideration the construction specifics of laser system. The method then displays anticipated results in compact form using equally sized matrices that have conditional formatting applied to their values. Present method allows for quick visual relation of parameters and their values as well as identification of patterns associated with the experiment (Figure 3).

The aim of the method is to make analysis of the colour laser marking experiments easier to understand, define, quantify, visualize, teach, and simulate by referencing it to existing and usually commonly accepted knowledge and formulas. The method offers a new way of looking at what actually happens to different experimental parameters before the actual marking of experiments. Therefore, as one identifies the set of the most important experimental parameters affecting the colour laser marking process, one can then better optimize the colour laser marking process itself.

The method implements one primary mathematical model of forecasting results for colour laser marking experiments that incorporates three secondary interconnected mathematical models (Figure 4). The primary mathematical model is a set of related matrices (Figure 5) with relations being defined by lasing formulas shown in Table I. The present method allows for addition of new as well as removal of unnecessary experimental parameter matrices, both technical and derived. The present method allows for very fast and flexible input of technical parameters and their values for the experiment as well as immediate output of the expected results. 


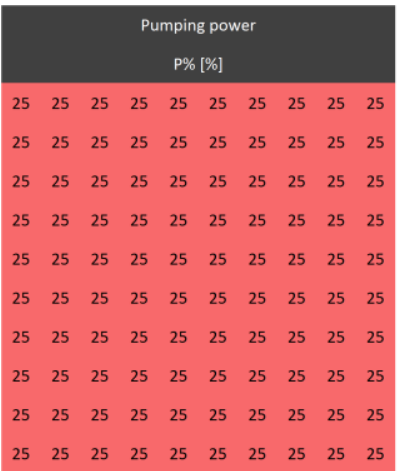

\section{Pulse overlap (linear pulse density $\mathrm{Ny}\left[\mathrm{mm}^{\wedge}-1\right]$ \\ 10000100000100001000001000011000010000010000010000110000} 8000800080008000800080008000800080008000 5000500050005000500050005000500050005000 4000400040004000400040004000400040004000 2500250025002500250025002500250025002500 2000200020002000200020002000200020002000 1600160016001600160016001600160016001600 1250125012501250125012501250125012501250 1000100010001000100010001000100010001000 800800800800800800800800800800

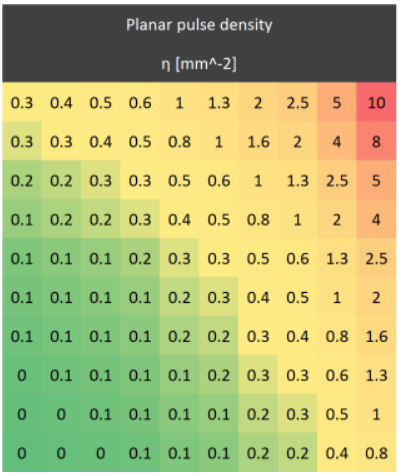

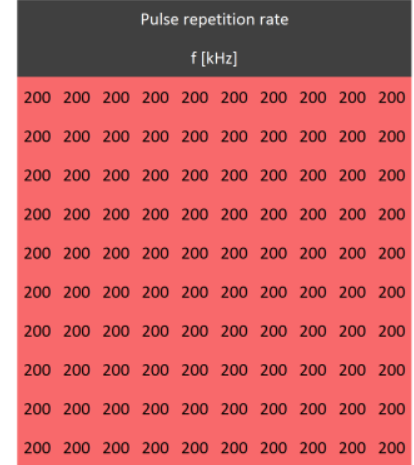
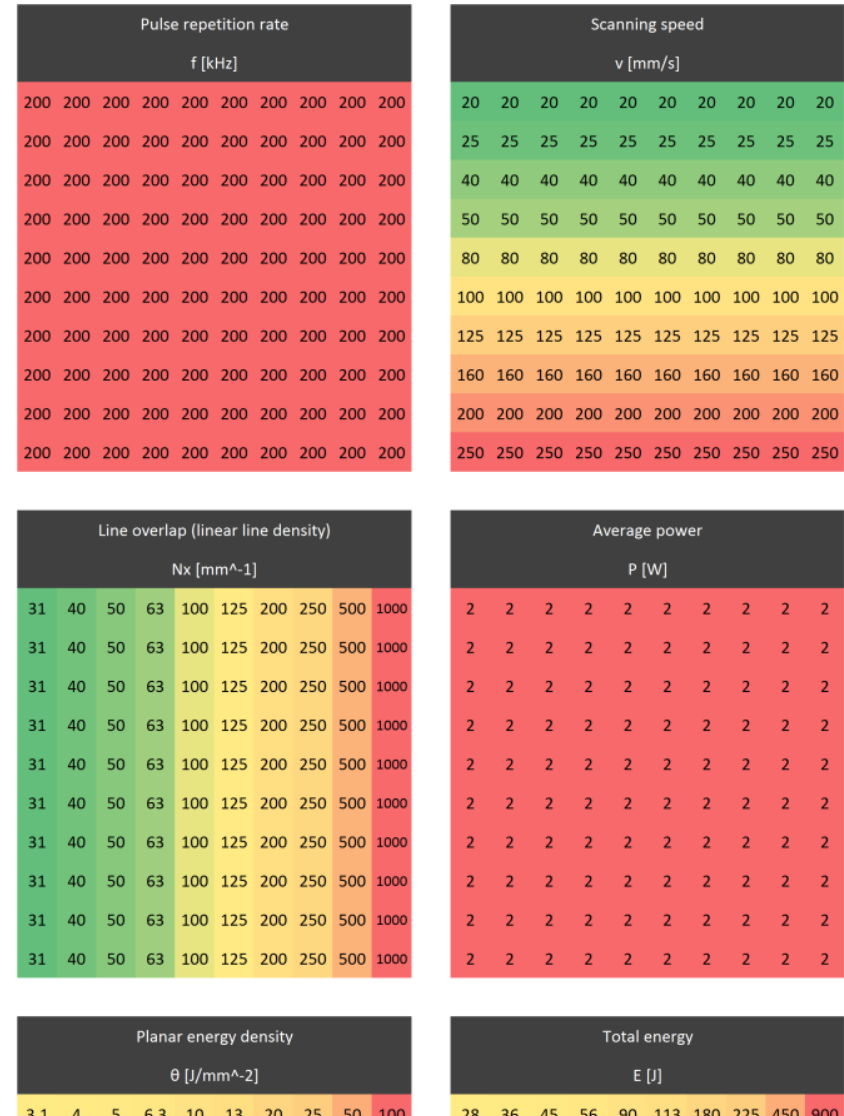

$\begin{array}{llllllllll}3.1 & 4 & 5 & 6.3 & 10 & 13 & 20 & 25 & 50 & 100\end{array}$ $\begin{array}{llllllllllll}2.5 & 3.2 & 4 & 5 & 8 & 10 & 16 & 20 & 40 & 80\end{array}$ $\begin{array}{llllllllll}1.6 & 2 & 2.5 & 3.1 & 5 & 6.3 & 10 & 13 & 25 & 50\end{array}$ $\begin{array}{llllllllll}1.3 & 1.6 & 2 & 2.5 & 4 & 5 & 8 & 10 & 20 & 40\end{array}$ $\begin{array}{llllllllllll}0.8 & 1 & 1.3 & 1.6 & 2.5 & 3.1 & 5 & 6.3 & 13 & 25\end{array}$ $\begin{array}{lllllllllll}0.6 & 0.8 & 1 & 1.3 & 2 & 2.5 & 4 & 5 & 10 & 20\end{array}$ $\begin{array}{lllllllllll}0.5 & 0.6 & 0.8 & 1 & 1.6 & 2 & 3.2 & 4 & 8 & 16\end{array}$ $\begin{array}{lllllllllll}0.4 & 0.5 & 0.6 & 0.8 & 1.3 & 1.6 & 2.5 & 3.1 & 6.3 & 13\end{array}$ $\begin{array}{lllllllllll}0.3 & 0.4 & 0.5 & 0.6 & 1 & 1.3 & 2 & 2.5 & 5 & 10\end{array}$ $\begin{array}{lllllllllll}0.3 & 0.3 & 0.4 & 0.5 & 0.8 & 1 & 1.6 & 2 & 4 & 8\end{array}$

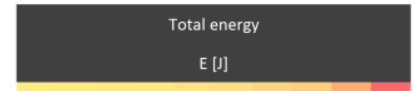

$\begin{array}{llllllllll}28 & 36 & 45 & 56 & 90 & 113 & 180 & 225 & 450 & 900\end{array}$ $\begin{array}{llllllllll}23 & 29 & 36 & 45 & 72 & 90 & 144 & 180 & 360 & 720\end{array}$ $\begin{array}{llllllllll}14 & 18 & 23 & 28 & 45 & 56 & 90 & 113 & 225 & 450\end{array}$ $\begin{array}{llllllllll}11 & 14 & 18 & 23 & 36 & 45 & 72 & 90 & 180 & 360\end{array}$ $\begin{array}{lllllllllll}7 & 9 & 11 & 14 & 23 & 28 & 45 & 56 & 113 & 225\end{array}$ $\begin{array}{lllllllllll}5.6 & 7.2 & 9 & 11 & 18 & 23 & 36 & 45 & 90 & 180\end{array}$ $\begin{array}{lllllllllll}4.5 & 5.8 & 7.2 & 9 & 14 & 18 & 29 & 36 & 72 & 144\end{array}$ $\begin{array}{lllllllllll}3.5 & 4.5 & 5.6 & 7 & 11 & 14 & 23 & 28 & 56 & 113\end{array}$ $\begin{array}{lllllllllll}2.8 & 3.6 & 4.5 & 5.6 & 9 & 11 & 18 & 23 & 45 & 90\end{array}$ $\begin{array}{llllllllllll}2.3 & 2.9 & 3.6 & 4.5 & 7.2 & 9 & 14 & 18 & 36 & 72\end{array}$
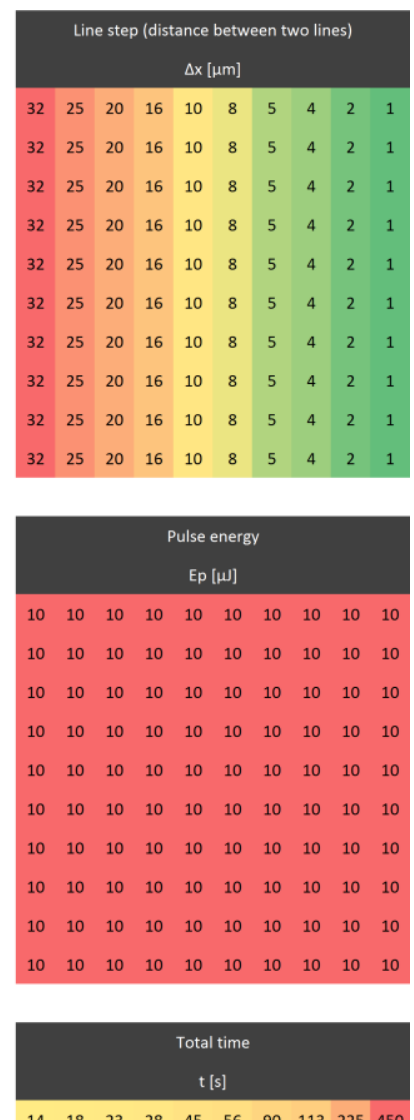

$\begin{array}{llllllllll}14 & 18 & 23 & 28 & 45 & 56 & 90 & 113 & 225 & 450\end{array}$ $\begin{array}{llllllllll}11 & 14 & 18 & 23 & 36 & 45 & 72 & 90 & 180 & 360\end{array}$ $\begin{array}{lllllllllll}7 & 9 & 11 & 14 & 23 & 28 & 45 & 56 & 113 & 225\end{array}$ $\begin{array}{llllllllll}5.6 & 7.2 & 9 & 11 & 18 & 23 & 36 & 45 & 90 & 180\end{array}$ $\begin{array}{lllllllllll}3.5 & 4.5 & 5.6 & 7 & 11 & 14 & 23 & 28 & 56 & 113\end{array}$ $\begin{array}{llllllllll}2.8 & 3.6 & 4.5 & 5.6 & 9 & 11 & 18 & 23 & 45 & 90\end{array}$ $\begin{array}{lllllllllll}2.3 & 2.9 & 3.6 & 4.5 & 7.2 & 9 & 14 & 18 & 36 & 72\end{array}$ $\begin{array}{lllllllllll}1.8 & 2.3 & 2.8 & 3.5 & 5.6 & 7 & 11 & 14 & 28 & 56\end{array}$ $\begin{array}{llllllllllll}1.4 & 1.8 & 2.3 & 2.8 & 4.5 & 5.6 & 9 & 11 & 23 & 45\end{array}$ $\begin{array}{lllllllllll}1.1 & 1.4 & 1.8 & 2.3 & 3.6 & 4.5 & 7.2 & 9 & 18 & 36\end{array}$

Figure 3. Overview of proposed mathematical model for experimentally produced sample (with constant pulse width of $4 \mathrm{~ns}$ ).

Once all three secondary mathematical models are defined, the primary mathematical model is set and can be instantiated for any different experiment. Thus, before one proceeds with actual marking of test matrices, it is possible to efficiently plan the experiments and see expected distribution of values of output parameters in advance.

\section{Results and discussion}

The method maps and visualizes parameters of colour laser marking experiments. The method displays all the necessary experimental parameters, both technical and derived, that are associated with marking experiment of a test matrix in a compact form of a set of equally sized matrices where each such matrix is assigned its own experimental parameter and stores that parameter's values positionally related to elements of test matrix to be marked as well as the values of every other experimental parameter matrix. Thus the number of rows and columns of each such experimental parameter matrix is identical to those of test matrix itself.

The method of mapping values of necessary experimental parameters within their own matrices to elements of the test matrix lets one immediately start seeing experimental values in context of two dimensions corresponding to those of test matrix, also in the context of values belonging to all other experimental parameter matrices, and, more importantly, in the context of marking colours to be produced. Thus one might think of the set of all experimental parameter matrices including the test matrix to be marked itself as a three-dimensional stack of matrices where each matrix cell that corresponds to specific row and specific column is directly associated with every other such cell up and down the stack. 


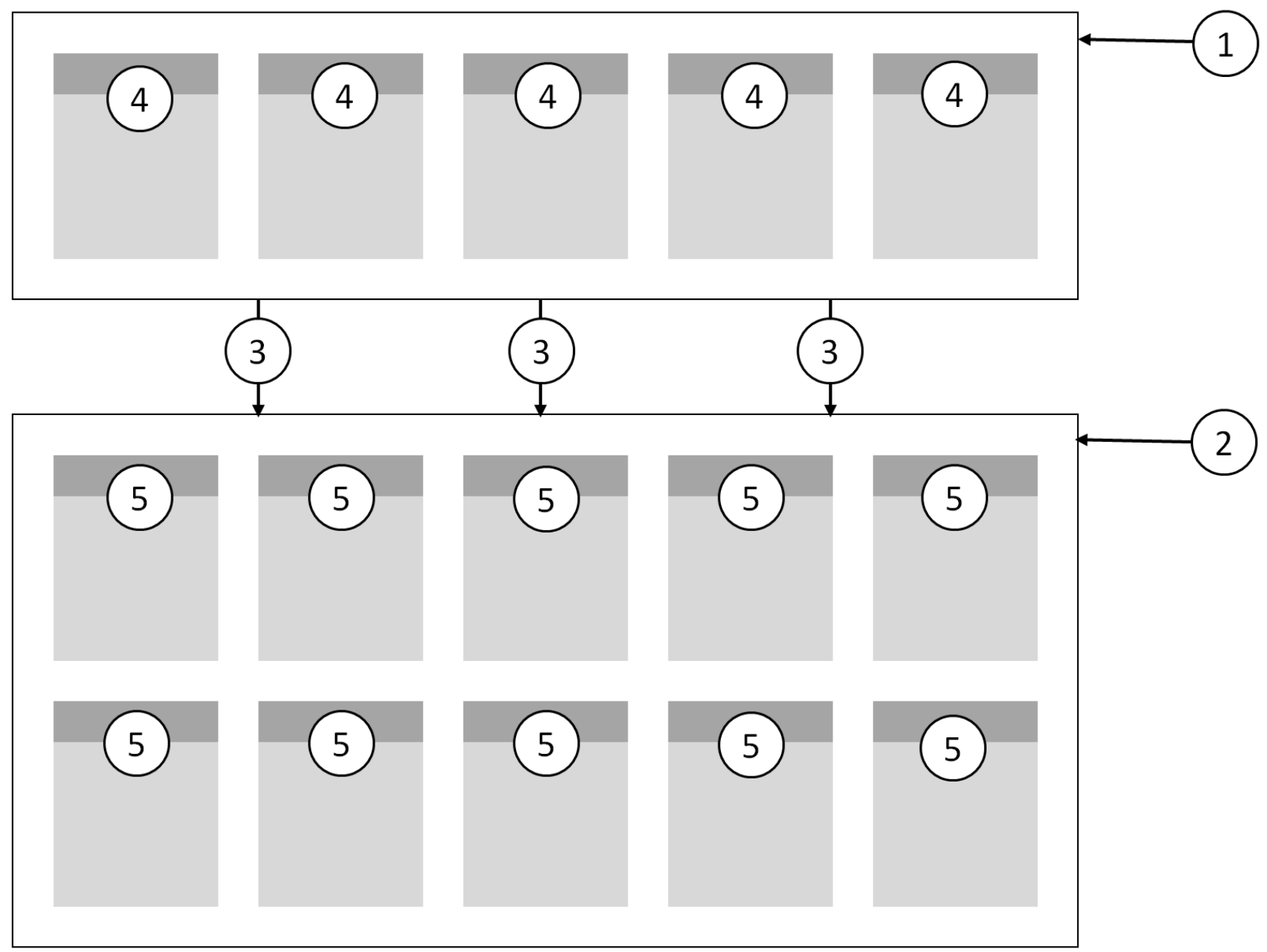

Figure 4. Schematic view of primary mathematical model implemented by the present method: (1) secondary mathematical model of input of experimental data; (2) secondary mathematical model of output of experimental data; (3) secondary mathematical model of laser system's construction specifics; (4) instances of laser system's technical parameter matrices; (5) instances of derived parameter matrices.

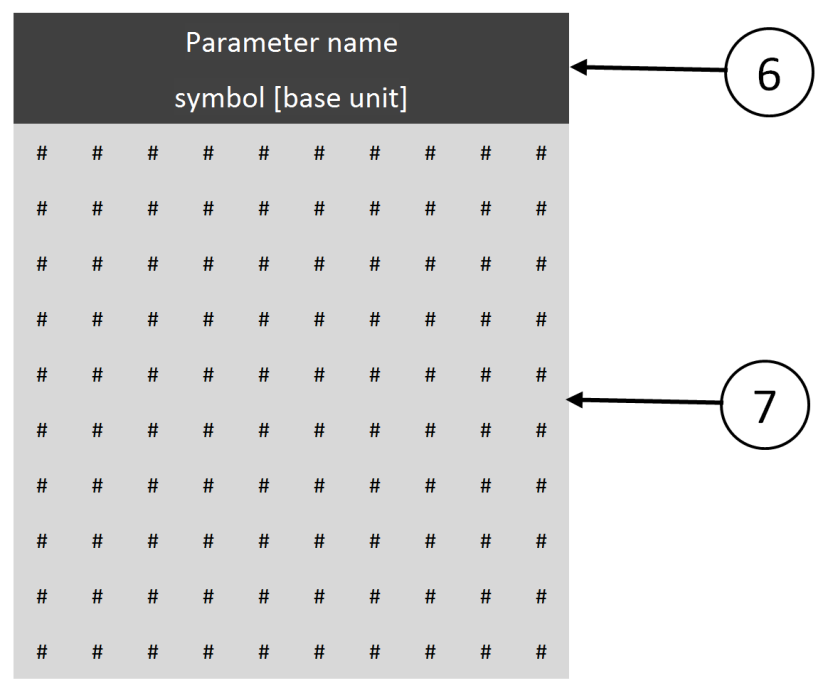

Figure 5. Schematic view of parameter's matrix: (6) header associated with parameter's matrix containing information about the parameter in question, its commonly accepted physical symbol and base unit; (7) two-dimensional array of distribution of values of the parameter.
By applying spreadsheet processor conditional formatting feature to the values of each experimental parameter matrix, one can see the planar distribution of relative magnitudes of these values coded with a colour scale. Thus each experimental parameter matrix becomes a colour map and can be analyzed when spreadsheet processor worksheet is zoomed out. Each such colour map helps efficiently spot contrasting values within all matrices just by quickly looking through them.

Interestingly enough, distinct patterns may emerge within some of such experimental parameter colour maps. Such patterns may even sometimes resemble the pattern seen within the marked test matrix itself. Though, more often similar patterns can be observed within the set of experimental parameter matrices. Thus if any two such patterns look similar or even identical one might assume these are somehow proportional to each other.

The method implements the primary mathematical model of forecasting results for colour laser marking experiments which in turn comprises three secondary 
interconnected mathematical models. The secondary mathematical model of laser system construction specifics allows user to define any conditions where laser system may behave differently by setting specific relations between the set of technical parameter matrices and the set of derived parameter matrices. The set of all laser system's technical parameter matrices represents the secondary mathematical model for input of experimental data. The set of all derived parameter matrices forms the secondary mathematical model for output of experimental data.

Thus the method allows for modelling distribution of the values within experimental laser parameter matrices for any particular colour laser marking experiment. Both secondary mathematical models for input of experimental data and for output of experimental data operate with numeric data.

\section{Conclusions}

The user's overall understanding of how the present method's primary mathematical model of forecasting laser marking experiment results works can help the user extract even more useful information out of it. Therefore the method can be used for interactive teaching purposes or to assist advanced users. Finally, the method can help its users better interpret and test common lasing formulas as well as produce new ones, and no similar modelling method, which allows that, yet exists.

There is always a problem of experiment repeatability when testing produced marking colours for technical parameters mentioned in research papers of other authors. The proposed mathematical model should be able to help authors to present their experimental parameters and their values as well as produced marking colours in a very compact form that is both easy to understand and easy to implement.

During testing of the mathematical model it was found that different formulas which define relations between the technical parameter set and the derived parameter set are easy to view, edit and copy. The method can also be useful for researchers testing different theories about colour laser marking, since adding new or eliminating unnecessary parameter matrices is easy and different emerging colour patterns help quickly spot parameters that matter in the context of colour laser marking experiments.

\section{Acknowledgements}

This research was supported by laser system equipment from Rofin-Sinar Laser GmbH.

\section{References}

E. H. Amara, F. Haïd, and A. Noukaz. Experimental investigations on fiber laser color marking of steels. Applied Surface Science, 351:1-12, 2015. doi: 10.1016/j.apsusc.2015.05.095

A. J. Antonczak, D. Kocoń, M. Nowak, P. Kozioł, and K. M. Abramski. Laser-induced colour marking-Sensitivity scaling for a stainless steel. Applied Surface Science, 264:229-236, 2013. doi: 10.1016/j.apsusc.2012.09.178

A. J. Antonczak, B. Stepak, P. E. Kozioł, and K. M. Abramski. The influence of process parameters on the laser-induced coloring of titanium. Applied Physics A, 115(3):1003-1013, 2014. doi: 10.1007/s00339-013-7932-8

J. Bliedtner, H. Muller, and A. Barz. Lasermaterialbearbeitung. Fachbuchverlag Leipzig im Carl Hanser Verlag, 2013. doi: 10.3139/9783446429291

J. Eichler and H. J. Eichler. Laser. Springer-Verlag Berlin, 1998. doi: 10.1007/978-3-662-08247-8

P. Laakso, H. Pantsar, and V. Mehtälä. Marking decorative features to stainless steel with fiber laser. IMD/ALAC, 2008.

A. Lehmuskero, V. Kontturi, J. Hiltunen, and M. Kuittinen. Modeling of laser-colored stainless steel surfaces by color pixels. Applied Physics B, 98(2-3):497-500, 2010. doi: 10.1007/s00340-009-3734-2

J. Qi, K. L. Wang, and Y. M. Zhu. A study on the laser marking process of stainless steel. Journal of Materials Processing Technology, 139(1-3):273-276, 2003. doi: 10.1016/S0924-0136(03)00234-6

V. Veiko, G. Odintsova, E. Ageev, Y. Karlagina, A. Loginov, A. Skuratova, and E. Gorbunova. Controlled oxide films formation by nanosecond laser pulses for color marking. Optics Express, 22(20):24342-24347, 2014. doi: 10.1364/oe.22.024342

V. Veiko, G. Odintsova, E. Gorbunova, E. Ageev, A. Shimko, Y. Karlagina, and Y. Andreeva. Development of complete color palette based on spectrophotometric measurements of steel oxidation results for enhancement of color laser marking technology. Materials \& Design, 89:684-688, 2016. doi: 10.1016/j.matdes.2015.10.030 